\title{
ELECTRICAL NERVE STIMULATION IN TRAUMA
}

\section{IMPRESSIONS ON (a) DIAGNOSTIC TESTS (b) THERAPY}

AS EXPERIENCED IN SOUTH AFRICA

By Miss J. BLAIR, M.C.S.P., T.C.S.P.

Senior Lecturer of Physiotherapy of the University of the Witwatersrand.

Paper delivered at the Third International Congress of the World Confederation of Physical Therapy in Paris, Sept. 1959.

Economically, as has been proved elsewhere, particularly in such rehabilitation centres as exist in America and the United Kingdom, physiotherapy has a valuable contribution to make to South African industry. In Johannesburg and on the Witwatersrand alone, the development of industry is a tremendous and ever-increasing programme.

It is interesting to note recent statistics made available by the Medical Consultant to the Anglo American Corporation of South Africa Limited-a mining group controlling twenty-one African and five European hospitals. In the Orange Free State where African hospitals are modern and Physiotherapy freely available, of 44,000 Africans employed at risk 50 are hospitalised and spend an average of $9 \frac{1}{2}$ days in hospital; on discharge $98 \%-99 \%$ are fit for underground duty. On the Witwatersrand where physiotherapy is not so widely used, of 15,000 Africans employed at risk 50 are hospitalised and spend an average of $7 \frac{1}{2}$ days in hospital; on discharge $10 \%$ are fit for underground duty, the remaining $80 \%$ spending from $2-3$ weeks on surface work and light duties. The authorities consider these figures as significant in proving the value of physiotherapy. Of these surgical cases a large proportion of them are in the traumatic neurological group.

The experiences noted in this paper do not deal with the Mine hospitals but are founded on cases treated in the Johannesburg General Hospital Group and Baragwanath Hospital. These two units are the teaching hospitals for the University of the Witwatersrand Medical School, and are divided as follows:

The Johannesburg General Hospital is the main hospital for the adult European population of Johannesburg and district-both medical and surgical. It has 600 beds and controls over 150 beds in four of the Johannesburg nursing homes where are housed special units, e.g. Surgical, Thoracic, Plastic, Neurosurgical and Geriatrics. Its subsidiary hospitals include the Transvaal Memorial Children's Hospital, with 200 beds, and the Queen Victoria Maternity Hospital, with 100 beds. Other hospitals in Johannesburg include Tara Hospital with 150 beds-a Psychiatric Unit for non-certifiable psychiatric patients, the Non-European Hospital with 100 beds - an emergency clearing centre for Africans, Asiatics and Coloureds, and Baragwanath Hospital with 1,800 beds-mainly for Africans

Physiotherapy Departments in these units, and also in most of the other larger centres in South Africa, are undoubtedly equipped to a higher standard than is general in Europe, and consequently physiotherapists have every opportunity and encouragement to improve accepted techniques and evolve and develop new ones.

At the Johannesburg General Hospital most of the peripheral and central nerve injuries are due to traffic accidents. In this country, as in many others, the traffic hazard is great. In addition, assaults (usually gunshots and stab wounds) account for a high proportion of the cases treated. Among the Africans the majority of peripheral nerve injuries are predominantly stab and knobkerry wounds, the reason being that in most instances the urban African has become de-tribalised and thus lives under unnatural conditions, often in overcrowded communities with few recreational facilities; due to the demands of industry. The authorities are doing much to improve the living conditions, but they cannot alter the African temperament, particularly that of the more primitive African.

There is also that colourfully sordid personality known as the 'Shebeen Queen'. A shebeen is a drinking den and the Queen is the woman who brews the drink, a violently alcoholic concoction often said to include carbide, metal polish, methylated spirits and dead rats, being known as 'Skokiaan' or 'Barberton'. It is simple to visualise the loss of inhibitions the imbibers have-knive, knobkerries, stilettos and even teeth, become lethal expressions of feelings.

We are fortunate in that, due to the co-operation and realisation of the value of physiotherapy by the medical staffs of our hospitals, these patients are seen by the physiotherapy team soon after admission or at a very early stage of their treatment; accordingly we are given the opportunity of minimising complications and prolonged after-care.

The Africans are divided into educated, urbanised and primitive categories, and among the majority of the more primitive Africans we encounter a special problem ... it has been observed that although he posseses great muscle strength in his trunk and limbs his skilled hand and finger( power is considerably less than that of the European. As a result it is possible to completely overlook weakness and paralysis particularly of the hands, fingers and lower leg. To him it is not a disability and his compensatory arm and leg strength overrides his local weakness; moreover, to this type of African his limbs are mere appendages and the loss of use of one limb can be made up by the remaining three. He is not apprehensive of injury by violence, or that which has visible signs, and in simple trauma will make a rapid and uncomplicated recovery.

Observations on Diagnostic Stimulation.

The Galvanic Faradic Test:

This quantitative method of testing has shown to be most inaccurate, as was proved by the Nerve Injuries Committee of the British Medical Research Council during the 1939-45 War. At our Non-European Hospital, for instance, in the presence of R.D. a normal response to faradism was elicited from 6 to 9 weeks following trauma to the nerve in approximately $30 \%$ to $50 \%$ of cases, and no response to faradism was elicited in cases where voluntary movement was present in recovering cases in over $50 \%$

The Accommodation Test: i.e. the capacity of a normal muscle to adapt itself and react to an electrical stimulus.

This in itself is found to be of little value, but corroborated by other evidence is a guide to prognosis, particularly in neuropraxia.

Intensity Duration Curves

Our experiences in South Africa are that this test, in the hands of skilled operators, is highly accurate and provides valuable information on diagnosis and prognosis. We have found that it is most necessary to plot both the square wave and exponential (or fully triangular) wave curves, particularly as a prognostic sign. As experience has shown that there may be a normal or almost normal curve using square wave current but a partial reaction of degeneration is clearly evident by the kinks and irregularities in the exponential wave curve. This evidence has been confirmed by Electromyographical tests, when fibrillations were noted in such cases where the exponential or triangular wave form showed abnormality and the square wave form simulated the normal.

Another reason for our preference for this method is the case with which the $R$ heobase and Chronaxie can be read from the chart, thus a series of corroborated evidence.

We choose the constant current, high impedance output generator registering contraction in current with continuously controlled pulse and pause durations with no fixed steps, because of the counter-action of the skin resistance factor, its greater accuracy, and its reduction of variables. Also with its continuously independent control of the pulse pause ratio, a more comprehensive graph can be obtained. 


\section{Observations on Therapy:}

In treatment wherever possible we favour the selective group technique rather than other methods in use, as we find it more comprehensive, physiologically more normal and less fatiguing to the muscles being stimulated.

By predetermining the pulse duration and alpha or current peak angle to which the paresed muscles respond best, that is without contraction overlay of unaffected muscles, and adapting the pause duration to minimise fatigue, a more effective treatment can be given, better records kept and less variables need be considered.

The more important variables that influence records of progress being: (a) the exact positioning of electrodes, (b) the order in which muscles are stimulated, (c) local skin temperature, and (d) the relative change in skin resistance.

It is also important that this method of treatment is far less time-consuming. For example if ten muscles were paralysed and individual stimulation were to be given effectively at a minimum period of five minutes per muscle, stimulation would be fifty minutes; in group stimulation all muscles would be treated together and a total time of five minutes would be necessary, thus reducing treatment time by 45 minutes.

It is important to maintain as strict a regime in therapy as it is in diagn sstic procedures and the full co-operation of the pa ient must be the first consideration. As I mentioned earlier, a mere paralysis, particularly if not gross, is not a disability to many of the Africans, unless it interferes with their earning capacity or dancing, which could well happen through early neglect of the condition, but it is often possibie to gain their co-operation by capturing their imagination; to see their muscle contraction by means outside their own eontrol or volition is 'white magic' to them.

We find that frequency of the treatment is dependent on severity, fatigue and the intelligent co-operation of the patient.

Pre-medication is well worth the trouble and by preheating and lowering skin temperature resistance treatment is greatly facilitated and more comfortable; to achieve this, moist heat is the preferable mediun.

Meticulous records of pulse, pause duration, alpha, fatigue time and current peak reading, together with timely progressing in application, is essential for early recovering and good results.

Also the conservative use of stimulation in conjunction with other forms of treatment.

\section{Conclusions}

It has become increasingly more evident that the introduction of exponential wave form current and the greater accuracy and wider range of square wave form in phy: io. therapy treatment units have done much to produce more accurate measures of diagnosis and more effective means of treatment.

In South Africa research and investigation into the treatment of trauma, particularly in that of Africans, is a fascinating and great challenge and one that the World Health Organisation might consider in the future.

\section{a mea spromeh to vasodilatation}

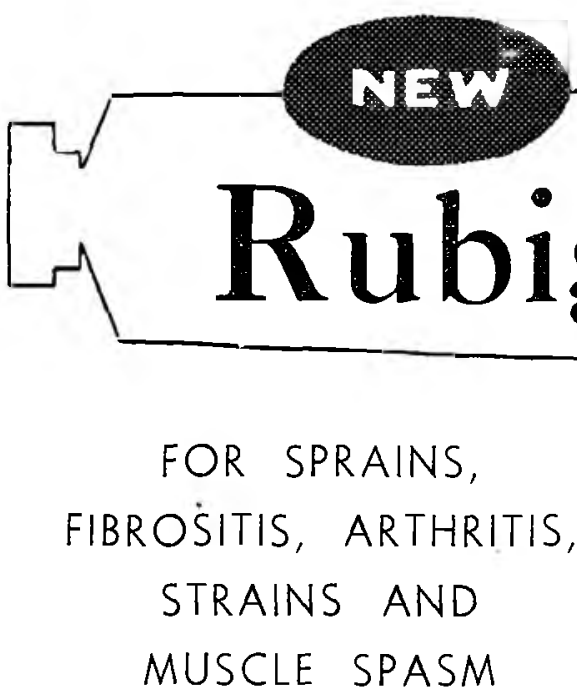

Registered User of Trade Mark and Distributors in South Africa:

\section{WYETH LABORATORIES (PTY.) LTD. 54, STATION STREET,}

by the use of

the potent new penetrative

\section{agent}

\section{METHYL NICOTINATE}

in conjunction with the powerful vasodilator, histamine opens the way for the histamine to penetrate tissues rapidly.

There it promotes prolonged, pain-relieving hyperemia, comforting analgesia and soothing warmth.

Formula

Histamine Dihydrochloride

Methyl Nicotinate

Glycol Monosalicylate

Capsicum Oleoresin

$0 \cdot 1 \%$

$1.0 \%$

$10.0 \%$
$0.1 \%$

These are incorporated in a bland, cosmetically pleasant vehicle.

How Supplied-Tubes of Price-Inexpensive - 5/6 One Ounce. per tube to your patient. 